\title{
Placenta percreta complicated by haemorrhagic shock and peripartum cardiomyopathy: A diagnostic as well as therapeutic dilemma
}

\author{
Kumar Rajani Kant ${ }^{1}$, B. Naveen Naik ${ }^{2}$, Kamal Kajal ${ }^{3 *}$, Shiv Lal Soni ${ }^{4}$ \\ ${ }^{1}$ Senior Resident, ${ }^{2-4}$ Assistant Professor, Dept. of Anaesthesia, ${ }^{1,3,4}$ Post Graduate Institute of Medical Education \& Research, Chandigarh, \\ ${ }^{2}$ Jawaharlal Institute of Postgraduate Medical Education and Research, Puducherry, India \\ *Corresponding Author: Kamal Kajal \\ Email:kamal.kajal@gmail.com
}

Received: $20^{\text {th }}$ January, 2019

Accepted: $25^{\text {th }}$ February, 2019

\begin{abstract}
Peripartum cardiomyopathy (PPCM) is a rare disease that usually manifests during late pregnancy or immediately after delivery. Cardiomyopathies are a heterogenous group of disorders affecting myocardium and characterized by low ejection fraction of heart leading to significant morbidity and mortality. Anaesthetic management of these patients for complex surgeries is challenging due to relatively fixed cardiac output, limited ability of myocardium to respond to inotropic drugs because of pre-existing higher sympathetic tone and lastly these patients are more prone for subsequent heart failure and pulmonary edema. These problems are further amplified in peripartum cardiomyopathy as a result of physiological cardiovascular changes in pregnancy and labour. We report a successful anaesthetic management of a patient with placenta percreta in haemorrhagic shock with associated deep venous thrombosis posted for emergency exploratory laparotomy, complicated by peripartum cardiomyopathy.
\end{abstract}

Keywords: Peripartum, Placenta percreta, Cardiomyopathy.

\section{Introduction}

An increasing trend of caesarean deliveries worldwide have led to a surge in cases of morbidly adherent placentation in subsequent pregnancies. Various types of variations in placenta are as follows. ${ }^{1}$

\begin{tabular}{|l|c|l|}
\hline Placenta accreta & $75-78 \%$ & $\begin{array}{l}\text { The placenta attaches strongly to } \\
\text { the myometrium, but does not } \\
\text { penetrate it. This form of the } \\
\text { condition accounts for around } \\
75 \% \text { of all cases. }\end{array}$ \\
\hline Placenta increta & $17 \%$ & $\begin{array}{l}\text { Occurs when the placenta } \\
\text { penetrates the myometrium. }\end{array}$ \\
\hline Placenta percreta & $5-7 \%$ & $\begin{array}{l}\text { The worst form of the condition } \\
\text { is when the placenta penetrates } \\
\text { the entire myometrium to the } \\
\text { uterine serosa (invades through } \\
\text { entire uterine wall). This variant } \\
\text { can lead to the placenta attaching } \\
\text { to other organs such as the } \\
\text { rectum or urinary bladder. }\end{array}$ \\
\hline
\end{tabular}

The management of such patients heralds significant challenges due to massive blood loss, disseminated intravascular coagulation and multi-organ dysfunction. In addition, co-existing obstetric conditions may be masked at the time of presentation but worsen an already compromised state leading to maternal morbidity and mortality.

Cardiomyopathies are a heterogenous group of myocardial diseases associated with mechanical or electrical discomfort. $^{2}$ They are either confined to heart or part of generalised systemic disorders. Hypertrophic cardiomyopathy (HOCM) is the most common genetic cardiomyopathy with incidence of 1 in 500 and transmitted as autosomal dominant trait. It is Characterized by LV hypertrophy and symptoms of angina, fatigue and heart failure. ${ }^{2}$ Dilated cardiomyopathy characterized by LV dilation, systolic dysfunction and normal LV wall thickness is transmitted as autosomal dominant. Restrictive cardiomyopathies are due to systemic diseases and are characterized by myocardial infiltration and severe diastolic dysfunction.

Peripartum cardiomyopathy (PPCM) has an incidence of 1 in $3000-4500$ live birth and develops from third trimester of pregnancy until five months after delivery. ${ }^{2,3}$ It develops as dilated form and occurs in women with no history of heart disease. Mortality rate ranges from 25 to $50 \%$ with most deaths occurring within 3 months. ${ }^{4}$ In this case of morbidly adherent placenta, the persistence of haemodynamic instability in the postpartum period posed significant challenge to anaesthesiologist due to underlying covert peripartum cardiomyopathy.

\section{Case Report}

A 32 year old female patient (weight $50 \mathrm{~kg}$, height $165 \mathrm{~cm}$ ) of gravida 4, para 3 with previous two LSCSs was operated outside in a private hospital for LSCS, baby delivered but placenta was found to be adherent to uterus, so it was partially removed and subsequently on $4^{\text {th }}$ postoperative day was referred to our institute in state of hypovolemic shock. Radiological imaging using CT scan of lower abdomen showed presence of placental tissue invading bladder wall. (Fig. 1) Deep vein thrombosis was diagnosed clinically based on swollen legs and confirmed by colourdoppler ultrasonography. Anticoagulants were not started due to the presence of hematuria and ongoing paravaginal bleed. Inferior Vena Cava filter was placed in IVC to prevent the possibility of pulmonary embolism. Bilateral uterine artery embolization was done to arrest ongoing bleeding. However, 
in view of persistent paravaginal bleeding and hematuria, patient was taken up for emergency exploratory laparotomy.

On preoperative assessment cardiorespiratory system was normal. Laboratory investigations showed $\mathrm{Hb}-8 \mathrm{gm} \%$, TLC-14200, platelet count-1.4 lakhs. Renal function tests, coagulogram profile, chest X-ray, ECG and ECHO were within normal limits. On arrival to operation theatre, all ASA standard monitors were attached. Baseline pulse rate and blood pressure were $105 / \mathrm{min}$ and $100 / 60 \mathrm{~mm}$ of $\mathrm{Hg}$ respectively. Arterial line in left radial artery and central line catheterization in right internal jugular vein were secured before anaesthesia induction for haemodynamic monitoring and fluid management. Patient was co-loaded with iv fluid ringers lactate and anaesthesia was induced with inj fentanyl $150 \mu \mathrm{g}$, inj thiopentone $200 \mathrm{mg}$, and Inj atracurium $25 \mathrm{mg}$. After endotracheal tube insertion anaesthesia was maintained with sevoflurane MAC of $0.6-1$ and $100 \% \mathrm{FiO}$. Goal directed fluid therapy was administered based on SPV, PPV and blood lactate levels. Intraoperatively patient had approximately six litres of blood loss calculated by swab weighing technique and drop in haemoglobin was verified by serial measurements of haematocrit values. Surgical duration lasted for $7 \mathrm{hrs}$ and patient received massive transfusion of 12 units of packed RBCs, 4 units of FFP and 8 units of platelet concentrates, guided by real time coagulogram with sonoclot analysis (SCP 2, SIENCO INC). Nor-adrenaline infusion titrated to mean arterial blood pressure was started intraoperatively. In view of prolonged surgery, major blood loss with massive blood transfusion and presence of haemodynamic instability on inotropic support, patient was planned for elective ventilation and shifted to ICU for further management. In ICU, further two units of PRBCs and FFPs given under guidance of sonoclot analysis. Echocardiography was done which revealed global hypokinesia with an ejection fraction of $40-45 \%$ according to modified Simpson rule as per our ICU protocol in patients with shock. After 48 hours of ICU stay, patient experienced supraventricular tachycardia that progressed to ventricular fibrillation and haemodynamic instability. ${ }^{5}$ Managed according to ACLS protocol and reverted to normal sinus rhythm in five minutes. Repeat 2Dechocardiography by cardiologist disclosed global hypokinesia with EF of $25 \%-30 \%$ that was attributed to peripartum cardiomyopathy (PPCM). She was then put on tab amiodarone (200mg), tab ramipril $(5 \mathrm{mg})$, tab carvedilol $(3.125 \mathrm{mg})$, tab furosemide $(20 \mathrm{mg})$, tab aldactone $(50 \mathrm{mg})$ and inj enoxaparin, on once a daily basis. Serial echo was done for next five days that showed no improvement in ejection fraction. Patient haemodynamics were gradually improved and she was weaned off from ventilator, extubated and shifted to HDU. After 7 days, warfarin was started and she was discharged on oral medications.

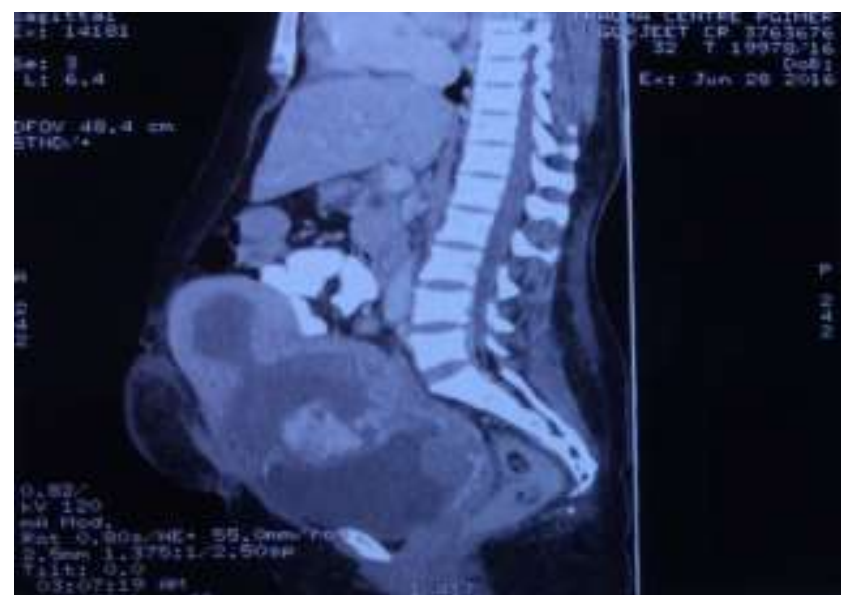

Fig. 1: CT scan of lower abdomen showing placenta penetrating myometrium and invading bladder

\section{Discussion}

Peripartum cardiomyopathy (PPCM) is defined by European Society of Cardiologists working group as "an idiopathic cardiomyopathy presenting with heart failure secondary to left ventricular systolic dysfunction towards the end of pregnancy or in the months following pregnancy where no other cause of heart failure is found. It is diagnosis of exclusion. The left ventricle may be dilated but ejection fraction is always nearly reduced below $45 \%$."

The disease is now known to have a multifactorial pathogenesis.

1. Myocarditis has been shown to be associated with PPCM although the incidence spans a wide range $(8.8-78 \%)^{6,7}$

2. Activation of autoimmune response has been cited as another causative factor. ${ }^{8,9}$

3. Another hypothesis postulated is that PPCM may be an abnormal cardiac response to hemodynamic changes associated with pregnancy. ${ }^{10}$

4. Other probable mechanisms proposed are an accelerated myocyte death (apoptosis), increase in proinflammatory cytokines, excessive prolactin production and coronary microangiopathy. ${ }^{11}$

In our case, preoperative echocardiography done by cardiologist at the time of IVC filter insertion was found to be normal. Hence, subsequent derangement in LV function postoperatively was quite remarkable and enforced us to search for etiology of the condition. Our major point of contention was whether decreased ejection fraction was due to myocardial damage suffered from stress or peripartum cardiomyopathy.

To reach conclusive diagnosis, troponin- $\mathrm{T}$ was done which was negative. No ECG changes were suggestive of myocardial ischaemia. Serial echocardiography was done postoperatively for 5 days and showed global hypokinesia with no improvement in left ventricular ejection fraction. Hence diagnosis of PPCM was made as a diagnosis of exclusion. 


\section{Conclusion}

Covert peripartum cardiomyopathy in a haemodynamically compromised patient with haemorrhagic shock, which later converted to overt cardiomyopathy subsequently postoperatively in ICU pose a significant diagnostic dilemma and challenge to anaesthesiologist. Point of care echocardiography along with evidence-based use of cardiac biomarkers should be done for optimal outcomes in critically ill parturients. Strong clinical suspicion and low threshold for diagnosis and timely therapeutic intervention can drastically improve prognosis in such patients. Multidisciplinary approach involving multiple specialties with effective communication and co-ordination can reduce both morbidity and mortality of parturients posted for emergency obstetric surgeries.

\section{Conflict of Interest: None.}

\section{References}

1. Steven G. Gabbe; Jennifer R. Niebyl; Joe Leigh Simpson, eds. (2002). Obstetrics: normal and problem pregnancies $\left(4^{\text {th }}\right.$ edition). New York, NY [u.a.]: Churchill Livingstone. p. 519.

2. Sliwa K, Hilfiker-Kleiner D, Petrie MC, Mebazaa A, Pieske B, Buchmann E, et al. Current state of knowledge on aetiology, diagnosis, management, and therapy of peripartum cardiomyopathy: A position statement from the Heart Failure Association of the European Society of Cardiology Working Group on peripartum cardiomyopathy. Eur J Heart Fail 2010;12:767-78.

3. Demakis JG, Rahimtoola SH, Sutton GC, Meadows WR, Szanto PB, Tobin JR, et al. Natural Course of Peripartum Cardiomyopathy. Circ 1971;44;1053-61.

4. Pandit V, Shetty S, Kumar A, Sagir A. Incidence and outcome of peripartum cardiomyopathy from a tertiary hospital in South India. Trop Doct 2009;39:168-9.
5. Gemici G, Tezcan H, Fak AS, Oktay A. Peripartum cardiomyopathy presenting with repetitive monomorphic ventricular tachycardia. Pacing Clin Electrophysiol 2004;27:557-8.

6. Midei MG, DeMent SH, Feldman AM, Hutchins GM, Baughman KL. Peripartum myocarditis and cardiomyopathy. Circ 1990;81:92.

7. Rizeq MN, Rickenbacher RR, Fowler MB, Billingham ME Incidence of myocarditis in peripartum cardiomyopathy. Am J Cardiol 1994;74:474-7.

8. Bultmann BD, Klingel K, Nabauer M, Wallwiener D, Kandolf R. High prevalence of viral genomes and inflammation in peripartum cardiomyopathy. Am J Obstet Gynecol 2005;193:363-5.

9. Warraich RS, Sliwa K, Damasceno A, Carraway R, Sundrom $\mathrm{B}$, Arif $\mathrm{G}$, et al. Impact of pregnancy-related heart failure on humoral immunity: Clinical relevance of G3-subclass immunoglobulins in peripartum cardiomyopathy. Am Heart J 2005;150(2):263-9.

10. Geva T, Mauer MB, Striker L, Kirshon B, Pivarnik JM. Effects of physiologic load of pregnancy on left ventricular contractility and remodelling. Am Heart J 1997;133:53-9.

11. Ntusi NB, Mayosi BM. Aetiology and risk factors of peripartum cardiomyopathy: A systematic review. Int $J$ Cardiol 2009;131:168-79.

How to cite this article: Kant KR, Naik BN, Kajal K, Soni SL. Placenta percreta complicated by haemorrhagic shock and peripartum cardiomyopathy: A diagnostic as well as therapeutic dilemma. Indian $J$ Clin Anaesth 2019;6(2):307-9. 